Ebisu Ebisu

Études japonaises Études japonaises

47 | printemps-été 2012

Catastrophes du 11 mars 2011, désastre de

Fukushima : fractures et émergences

\title{
L'empire du nucléaire : quand Fukushima ne crachait pas encore ses " atomes pour la paix »
}

The Nuclear Empire

at a Time When Fukushima Had Not Yet Split One "Atom For Peace"

原子力の帝国一福島がまだ「平和のための原子力」を吐出していな

かったと

Thierry Ribault

(2) OpenEdition

Journals

Édition électronique

URL : http://journals.openedition.org/ebisu/307

DOI : 10.4000/ebisu.307

ISSN : 2189-1893

Éditeur :

Institut français de recherche sur le Japon (UMIFRE 19 MAEE-CNRS), Maison franco-japonaise

Édition imprimée

Date de publication : 1 juin 2012

Pagination : 89-98

ISSN : 1340-3656

Référence électronique

Thierry Ribault, «L'empire du nucléaire : quand Fukushima ne crachait pas encore ses « atomes pour la paix » », Ebisu [En ligne], 47 | printemps-été 2012, mis en ligne le 03 avril 2014, consulté le 20 avril 2019. URL : http://journals.openedition.org/ebisu/307 ; DOI : 10.4000/ebisu.307 


\section{L'empire du nucléaire \\ Quand Fukushima ne crachait pas encore ses « atomes pour la paix»}

Thierry RIBAULT

Des vertus des souverains, la plus salutaire, dit-on, est : bien oublier*.

Nucléaires civil et militaire sont les deux faces d'une même pièce, et le reste n'est que propos lénifiants tenus par des pseudologues ayant perdu de vue la limite entre le vrai et le faux. Faire accepter le premier est une étape importante pour prolonger le développement du second. Dans cette perspective, les États-Unis ont utilisé le Japon pour perfectionner leurs technologies. À moins que le Japon n'ait, lui aussi, utilisé les États-Unis afin, le moment voulu, d'être autonome militairement et d'avoir une force de frappe nucléaire à disposition. Ce qui est certain, c'est que la politique nucléaire japonaise est arrimée à la politique américaine et anticommuniste ; cela permet de comprendre pourquoi l'un des pays les plus assujettis aux menaces sismiques est devenu la troisième nation nucléaire du monde. Cette situation cesse de sembler paradoxale dès lors qu'on prend en considération le fait que le développement du nucléaire civil au Japon est une émanation du nucléaire militaire américain. Il s’inscrit, de fait, dans la propagation de la doctrine soutenue par le président Dwight D. Eisenhower

\ Thierry Ribault, économiste, est chercheur au CNRS, à l'Institut français de recherche sur le Japon (UMIFRE 19 CNRS-MAEE), Maison franco-japonaise de Tokyo. Il a fait paraître, avec Nadine Ribault, Les Sanctuaires de l'abîme. Chronique du désastre de Fukushima, aux Éditions de l'Encyclopédie des Nuisances, Paris, 2012.

* Antigone, film de Jean-Marie Straub et Danièle Huillet, 1992. 
dans son discours "Atomes pour la paix ", prononcé le 8 décembre 1953 devant l'assemblée des Nations unies :

"Les États-Unis s'engagent devant vous et donc devant le monde à vouer intégralement leur cœur et leur esprit à la recherche d'une façon de permettre à l'homme de ne pas dédier sa miraculeuse inventivité à sa mort, mais de la consacrer à sa vie [...]. L'objectif de mon pays est d'aider à sortir de la chambre obscure des horreurs pour aller vers la lumière, pour trouver une manière de faire évoluer les esprits des hommes, les espoirs des hommes et les âmes des hommes d'où qu'ils soient, vers la paix, le bonheur et le bien-être. ”

Il s'agit donc de « livrer les secrets de l'atome aux mains de ceux qui sauront comment le dévêtir de son enveloppe militaire pour l'adapter aux arts de la paix » et de

«transformer les plus grandes forces de destruction en une formidable bénédiction, pour le bien-être de l'humanité toute entière [...]. Les États-Unis savent que la puissance de paix de l'énergie atomique n'est pas un rêve du futur. Son potentiel avéré existe déjà aujourd'hui. » (Hewlett \& Holl 2008 ; Cantelon 1992) ${ }^{1}$.

Quel est donc ce rêve du présent dans lequel le Japon s'est engouffré ? Un empire du nucléaire bâti sur deux préceptes nihilistes : le monde comme laboratoire d'une part, le désastre comme horizon de l'autre.

\section{Le monde comme laboratoire}

Le docteur Matsui Eisuke 松井英介 rappelait dans une allocution récente ${ }^{2}$ que l'Atomic Bomb Casualty Commission (ABCC), créée en 1946 par le président Truman, chargée de collecter les données relatives aux effets du rayonnement et de mener des recherches sur les conséquences sanitaires des bombes atomiques de Hiroshima et de Nagasaki, n'a jamais contribué au soin des victimes des bombes mais a essentiellement servi de laboratoire expérimental au service de l'idéologie du progrès scientifique dans le domaine nucléaire, civil ou militaire. En 1947, quelques mois après la création de l'ABCC, est

1. Voir aussi <AtomicArchives.com>.

2. The International Conference of Citizens and Scientists, 12 octobre 2011, Tokyo. 
fondé le Japan National Institute of Health (JNIH) (YOKEN en japonais, abréviation de Kokuritsu yobō eisei kenkyūjo 国立予防衛生研究所) ${ }^{3}$, également sur ordre des autorités d'occupation américaine. Le personnel de cet institut sera soigneusement choisi parmi les anciens membres et les collaborateurs de l'Unité 731 (Nanasan.ichi butai 七三一部隊) de l'Armée impériale située à Harbin, en Chine. Dirigée par le lieutenant-général Ishii Shirō 石井四郎, l'Unité 731 avait été chargée entre 1937 et 1945 d'effectuer des tests secrets d'armes bactériologiques et chimiques sur des cobayes humains en Chine - entre 3000 et 10000 soldats et civils chinois - y compris sur des prisonniers de guerre américains. Grâce au pacte signé entre Douglas McArthur et Ishii Shirō, les responsables de ces expérimentations échapperont aux poursuites devant le Tribunal de Tokyo après la guerre, en échange des résultats obtenus. Ainsi, six des huit directeurs qui se sont succédés à la tête du JNIH entre 1947 et 1983, ont travaillé pour l'Unité 731. La JNIH a prolongé sa coopération durant vingt ans avec le Laboratoire médical 406 de l'armée américaine, structure en charge de la préparation à la guerre bactériologique située près de Tokyo, ainsi qu'avec l'ABCC. Selon le professeur Shibata Shingo 柴田慎吾 (Lanouette \& von Hippel, 1990), de l'université de Hiroshima, les données récupérées par l'ABCC et le JNIH sur les victimes des radiations ont été uniquement utilisées pour moderniser les armes et les centrales nucléaires. Les autorités japonaises et américaines de l'époque ont demandé à la JNIH et à l'ABCC de coopérer afin de contraindre les victimes des bombes atomiques de participer aux études sur la radiation. Ainsi, selon Kojima Saburō 小島三郎, l'un des vice-directeurs du JNIH, ancien professeur à l'université de Tokyo, ayant servi dans l'Unité 731 : « Nous, les scientifiques intelligents, avions tous considéré que nous ne pouvions manquer cette opportunité en or d'étudier les effets du rayonnement atomique sur les humains » (Harris 2003). Les échantillons de sang prélevés par l'ABCC étaient ainsi directement fournis aux chercheurs du JNIH, ce dernier exerçant des pressions auprès des familles des défunts pour qu'elles autorisent des autopsies sur les cadavres de leurs proches. En avril 1975, l'ABCC a été remplacée par la Radiation Effects Research Foundation (RERF) (Zaidan

3. Le $1^{\text {er }}$ avril 1997 , face aux pressions de l'opinion publique quant à son passé sulfureux, le ministère de la Santé rebaptisera le JNIH en " National Institute of Infectious Diseases » (NIID) (Kokuritsu kansenshō kenkyūjo 国立感染症研究所). 
hōjin Hōshasen eikyō kenkyūjo 財団法人放射線影響研究所). La RERF, très activement impliquée dans l'enquête de santé publique qu'ont lancée les autorités à Fukushima dès juin 2011, n'est pas elle-même sans lien avec la Nippon Foundation (Nippon zaidan 日本財団), grand argentier du négationnisme, qu'il soit historique ou nucléaire ${ }^{4}$. De fait, si l'on en croit les rapports d'activité de la RERF, la Sasakawa Memorial Health Foundation (Sasakawa kinen hoken kyōryoku zaidan 笹川記念保健協力財団) - une des multiples tentacules de la Nippon Foundation - finance des activités de recherche de la RERF depuis au moins les années 1990, notamment à travers le "Chernobyl Sasakawa Health and Medical Cooperation Project " entre 1991 et 2001. Parmi les bénéficiaires de ces fonds, on trouve notamment Shibata Yoshisada 柴田義貞, du département d'épidémiologie et de biométrie de l'université de Nagasaki, proche collègue de Yamashita Shun.ichi 山下俊一, endocrinologue histrionique qui recommande aujourd'hui à la population de Fukushima de sourire pour faire face aux radiations, ainsi que Shigematsu Itsuzō 重松逸造, président de la RERF. À la même époque, en 1995, un comité d'experts internationaux baptisé Blue Ribbon Panel a été appelé à examiner l'avenir de la RERF, doublement menacé par une crise budgétaire et par une rupture de contrat avec la National Academy of Sciences américaine. On trouve dans ce comité, et sur recommandation de la partie japonaise de la fondation, Dan Beninson, un physicien argentin membre puis directeur de la Comisión Nacional de Energía Atómica d'Argentine et président de commission à l'ICRP ${ }^{5}$. Ce conseiller de l'Académie pontificale des Sciences du Vatican ${ }^{6}$ était en outre le mentor d'Abel González, lui aussi membre de l'ICRP et négateur des conséquences sanitaires de Tchernobyl. Le Blue Ribbon Panel concluera que l'intérêt des travaux de la RERF justifie pleinement la prolongation des financements du Département de l'Énergie américain ; et un rapport établi en 2008 par un Senior Review Panel recommandera la prolongation, sur les

4. Sasakawa Ryōichi 笹川良一, fondateur de la Fondation Sasakawa, qui deviendra la Nippon Foundation, criminel de guerre de rang A non condamné, sera libéré en 1948.

5. International Commission on Radiological Protection.

6. La Nippon Foundation semble nourrir des liens étroits avec des scientifiques proches du Vatican : le mexicain Mario Molina, prix Nobel de chimie, membre de l'Académie pontificale des Sciences du Vatican, a reçu en 1999 le prix Sasakawa des Nations unies (La Gaceta, lundi 22 février 2010). 
vingt prochaines années, des études menées par la Fondation ${ }^{7}$. Nul doute que l'enquête de santé publique de Fukushima, menée auprès de 2 millions de personnes avec un suivi sur trente ans pour certaines populations, va fournir du grain à moudre d'une extrême fraîcheur à cette organisation généalogiquement gourmande en expérimentation grandeur nature.

\section{Le désastre comme horizon ${ }^{8}$}

En 1954, dans le prolongement logique du discours "Atomes pour la paix » prononcé par Eisenhower, le gouvernement japonais inaugure le financement de son premier programme de recherche nucléaire. La Loi fondamentale sur l'énergie atomique est votée en décembre 1955. Dans un souci de conformité avec l'article 9 de la Constitution japonaise de 1947, selon lequel le peuple japonais renonce à la guerre en tant que droit souverain de la nation ainsi qu'à tout potentiel militaire, la loi sur l'énergie atomique vise à " utiliser l'énergie atomique pour le bien-être de l'humanité et l'élévation du niveau de vie ». Dans son article 1 alinéa 2, elle stipule que " la recherche, le développement et l'utilisation de l'énergie nucléaire doivent être restreints à des objectifs pacifiques ", que " le programme nucléaire japonais doit viser la sûreté et doit être réalisé de manière indépendante sous administration démocratique ", et que " les résultats et les informations obtenus doivent être rendus publics afin de contribuer activement à la coopération internationale » (Kawabe 2008).

Les États-Unis comme le Japon trouveront en la personne de Shōriki Matsutarō 正力松太郎 un remarquable " coopérateur international ". Candidat à la mairie de Tokyo dans les années 1930, ancien préfet de police, président du quotidien Yomiuri shinbun 読売新聞, fondateur de l'équipe de baseball Yomiuri Giants et futur président du réseau de télévision Nippon Television (NTV), Shōriki a passé - lui aussi - deux ans à la prison de

7. D'après l'Office of Health, Safety and Security des États-Unis [http://hss.energy. gov/healthsafety/IHS/hstudies/japan_radiate.html].

8. Nous reprenons ici des éléments figurant dans l'ouvrage de Nadine et Thierry Ribault (2012). 
Sugamo en tant que criminel de guerre de rang A, d'où il sera relâché sans jugement deux ans plus tard. Il est alors placé en liberté surveillée, ce qui l'empêche notamment de participer à la vie politique et de travailler dans les médias. Mais ces restrictions seront vite levées lorsque la péninsule coréenne deviendra en 1950 le terrain d'affrontement entre les États-Unis et l'armée rouge chinoise. L'anticommunisme américain trouvera dans la montée de Mao Zedong en 1949 et le début de la guerre de Corée, un ferment fort efficace que ne manquera pas de cultiver, parmi bien d'autres, Karl Mundt, sénateur du Dakota du Sud, membre influent du « comité parlementaire sur les activités anti-américaines " présidé par Joseph McCarthy, et fondateur de l'instrument de propagande " ouverte " qu'est The Voice of America. Mundt cherche en effet, à partir de 1950, à développer des réseaux de télévision en Turquie, aux Philippines, en Indonésie et au Japon, et se rapproche de Shōriki Matsutarō, considéré, malgré (ou plutôt grâce à) son passé récent de criminel de guerre, comme un allié de choix étant donné son anticommunisme virulent et son ancienne ferveur pour la colonisation japonaise de la Mandchourie.

La "purge rouge " qui sévira au Japon entre 1948 et 1950 lui donnera

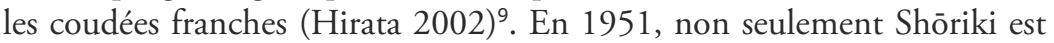
totalement blanchi - il ne sera pas le seul - mais on lui accorde la licence d'opérateur de télévision qui lui permet de fonder le réseau de diffusion Nippon Television Network (NTV). Outre Shōriki Matsutarō et Shibata Hidetoshi 柴田秀利, son secrétaire particulier, on compte parmi les promoteurs de NTV William Castle, ancien ambassadeur des États-Unis au Japon et consultant pour la CIA, Eugene Dooman, ancien membre de la CIA engagé dans la "guerre psychologique », James Kaufmann, avocat chez RCA et General Electric, professeur de droit occidental à l'université de Tokyo et consultant pour la CIA, James Murphy, ancien membre de l'agence de renseignement OSS et avocat pour NTV, et William Donovan, directeur de la même agence et conseiller pour le National Security Act de

9. Selon Hirata Tetsuo 平田哲男 (2002), entre décembre 1948 et novembre 1950, plus de 27000 personnes - fonctionnaires des administrations nationales et locales, syndicalistes, enseignants et salariés du privé - ont été licenciées parce qu'elles étaient considérées comme " rouges". 
$1947^{10}$. NTV est de fait, à l'époque, un outil de propagande américaine diffusant des programmes essentiellement fournis par la CIA et l'USIA (United States Information Agency) (Arima 2006).

Shōriki Matsutarō est élu député à la chambre basse de la Diète en février 1955, nommé ministre d'État pour l'Énergie atomique dans le cabinet de Hatoyama Ichirō 鳩山一郎 en décembre 1955, et devient premier président de la Commission nationale de l'énergie atomique établie par la Loi fondamentale précitée. Il est nommé en 1956 premier directeur de l'Agence pour la science et la technologie et se fait l'ardent promoteur de l'énergie nucléaire au Japon, en collaboration avec d'autres politiciens dont Nakasone Yasuhiro 中曽根康弘 ${ }^{11}$, à l'époque président du Comité à l'énergie nucléaire à la chambre basse, qui deviendra ministre de la Science en 1959 dans le cabinet de Kishi Nobusuke 岸信介 (autre ancien criminel de guerre de rang A, proche de Sasakawa Ryōichi), puis Premier ministre en 1982.

Dans le même temps, l'ambassade américaine, le Service d'information des États-Unis (USIS) et la CIA se lancent dans une vaste campagne de promotion de l'énergie nucléaire au Japon, campagne dont Shōriki Matsutarō sera l'un des piliers. En janvier 1955, le député américain Sidney Yates propose de construire la première centrale nucléaire japonaise à Hiroshima. Shōriki coorganise alors au Japon une exposition intitulée "L'usage pacifique de l'énergie nucléaire ». Le Yomiuri, journal à grand tirage qu'il dirige, soutient cette exposition qui promeut le retour de l'atome au Japon. Une cérémonie shintô de propitiation est organisée à Tokyo pour l'inauguration, le $1^{\text {er }}$ novembre 1955. L'ambassadeur américain y lit alors un message du président Eisenhower proclamant que cette exposition est « le symbole de la détermination mutuelle déployée par nos deux pays pour dédier désormais la grande puissance de l'atome aux arts de la paix " (Kuznick \& Gilbert 2010).

10. Cette loi promulguée le 26 juillet 1947, réorganisant les forces armées, la politique étrangère et les services de renseignement américains, fonde le Conseil de sécurité nationale et la CIA placés sous l'autorité du président des États-Unis.

11. Connu à l'époque pour avoir reproché à l'empereur Hirohito d'être responsable de la défaite du Japon. 
L'exposition dure six semaines à Tokyo, puis se transporte à Hiroshima et dans six autres villes. On dénombre 155000 visiteurs à Kyoto et 110000 à Hiroshima en trois semaines, dont de nombreux enfants en voyage scolaire. Tandis que dans les autres villes, l'exposition est sponsorisée exclusivement par le journal Yomiuri, elle a pour partenaires à Hiroshima la municipalité, le département, l'université et le journal Chügoku 中国新聞. Vingt personnes influentes dont le maire de Hiroshima, le président du département, le président de l'université et le président du journal Chügoku font partie du comité préparatoire, et se font les chantres de cette "nouvelle puissante énergie " (Tanaka \& Young 2010). Les mérites du "nucléaire pacifique " sont abondamment vantés, des applications médicales à l'électricité, en passant par la recherche, le contrôle des insectes et la conservation alimentaire. Sécurité, abondance et paix sont les mots d'ordre de cette grande messe technologique de la réconciliation avec le pire. Deux ans plus tard, la même exposition est de nouveau présentée à Hiroshima par la municipalité, cette fois au sein de la "Grande exposition de la reconstruction de Hiroshima ", visant à célébrer la renaissance de la ville détruite treize ans auparavant. Cinquante jours durant, à partir d'avril 1958, 917000 personnes défilent dans le pavillon dédié à "L'usage pacifique de l'énergie nucléaire " situé dans le Musée de la Bombe A, construit en 1955.

Les multiples campagnes de propagande menées conjointement par les autorités américaines et japonaises vont rapidement faire basculer l'opinion publique, en dépit des résistances liées au passé récent. Selon un rapport américain de l'USIS, $30 \%$ seulement des Japonais considèrent l'atome comme « nocif » en 1958 - contre 70 \% deux ans plus tôt (Osgood 2008).

Le Japon acquiert son premier réacteur nucléaire auprès du RoyaumeUni, avant de se tourner vers les fournisseurs américains. Au milieu de l'année 1957, est passée commande de vingt réacteurs supplémentaires. Kishi Nobusuke, précédemment évoqué, fervent défenseur d'une révision du traité de sécurité nippo-américain signé en 1951 et d'un arsenal «nucléaire de défense " pour le Japon, est alors Premier ministre.

En 1982, lors d'une audition devant un comité du Congrès américain ${ }^{12}$, l'amiral Hyman G. Rickover, artisan du prototype du réacteur Mark I

12. "On the Hazards of Nuclear Power. Testimony to Congress " (Rickover 1982). 
reproduit partout dans le monde, notamment au Japon ${ }^{13}$, ingénieur en chef du Nautilus, premier sous-marin américain à propulsion nucléaire et de la première centrale nucléaire américaine de Shippingport en Pennsylvanie, promoteur acharné de la prolifération de l'énergie nucléaire " civile ", icône du complexe militaro-industriel américain, répond à une question qui lui est posée concernant le bien-fondé du développement du nucléaire :

« Il y a deux milliards d'années, la vie n'existait pas sur la Terre à cause des radiations. Avec la puissance nucléaire, nous créons quelque chose que la nature a essayé de détruire pour rendre la vie possible... Chaque fois que vous produisez du rayonnement, vous produisez quelque chose qui a une demi-vie donnée, dans certains cas pour des millions d'années. Je crois que la race humaine va se naufrager elle-même, et il est fondamental que nous ayons le contrôle de cette force horrible et que nous essayons de l'éliminer... Je ne crois pas que la puissance nucléaire vaille la peine si elle génère du rayonnement. Alors vous allez me demander pourquoi j’ai développé des navires à propulsion nucléaire. C’est un mal nécessaire. Si ça ne tenait qu’à moi, je les coulerais tous... Ai-je répondu à votre question ?"

20 mai 2011

13. Cinq des six réacteurs de la centrale de Fukushima Daiichi sont de type Mark I. 


\section{Références}

ARIMA Tetsuo 有馬哲夫, 2006

Nihon Terebi to CIA : hakkutsu sareta «Shōrikifairu»日本テレビとCIA : 発掘され た「正カファイル」(Nihon TV et la CIA : les révélations du « dossier Shōriki »), Tokyo, Shinchōsha 新潮社.

CANTELON Philip L. (ed.), 1992 The American Atom: A Documentary History of Nuclear Policies from the Discovery of Fission to the Present, Philadelphia, University of Pennsylvania Press.

\section{HARRIS Sheldon H., 2003}

"Japanese Biomedical Experimentation During World War II Era ", in Military Medical Ethics, vol. 2., Department of Defense, Office of the Surgeon General, US Army, Borden Institute, Fort Detrick, 868 p.

HEWLETT Richard G. \& HOLL Jack M., 2008

Atoms for Peace and War, 1953-1961:

Eisenhower and the Atomic Energy Commission, American Council of Learned Societies, Humanities E-Book.

HIRATA Tetsuo 平田哲男, 2002 Reddo päji no shiteki kyūmei レッド・パ 一ジの史的究明 (Recherche historique sur la Purge rouge), Tokyo, Shin Nihon shuppansha 新日本出版社.

\section{KAWABE Ichiro, 2008}

« Nuclear Disarmament in Japan:

Background of the "Anti Anti-Nuclear Nation" », New Internationalist Japan, juin, $100: 38-52$.

\section{KUZNICK Peter J.}

\& GILBERT James (ed.), 2010

Rethinking Cold War Culture, Smithsonian Books, Washington DC.

\section{LANOUETTE William \& von HIPPEL}

\section{Frank, 1990}

"Biothech Lab Recalls Biowar», The Bulletin of the Atomic Scientists, janvierfévrier, p. 6.

\section{OSGOOD Kenneth, 2008}

Total Cold War. Eisenhower's Secret Propaganda Battle at Home and Abroad, University Press of Kansas, Lawrence.

RIBAULT Nadine et Thierry, 2012 Les Sanctuaires de l'abîme. Chronique du désastre de Fukushima, Paris, Éditions de I'Encyclopédie des Nuisances.

\section{RICKOVER Hyman G., 1982}

«On the Hazards of Nuclear Power. Testimony to Congress », 28 janvier 1982, in Economics of Defense Policy: Hearing before the Joint Economic Committee, Congress of the United States, $97^{\text {th }}$ Cong., $2^{\text {nd }}$ sess., Pt. 1.

TANAKA Yuki

\& YOUNG Marilyn B. (ed.), 2010 Bombing Civilians, A Twentieth-Century History, New York, The New Press. 